Relations industrielles

Industrial Relations

\title{
Immigrants in Industry, par Sheila Paterson, London, Oxford University Press, 1968, 425 pages.
}

\section{Pierre Brien}

Volume 24, numéro 3, 1969

URI : https://id.erudit.org/iderudit/028054ar

DOI : https://doi.org/10.7202/028054ar

Aller au sommaire du numéro

Éditeur(s)

Département des relations industrielles de l'Université Laval

ISSN

0034-379X (imprimé)

1703-8138 (numérique)

Découvrir la revue

Citer ce compte rendu

Brien, P. (1969). Compte rendu de [Immigrants in Industry, par Sheila Paterson, London, Oxford University Press, 1968, 425 pages.] Relations industrielles /

Industrial Relations, 24(3), 640-640. https://doi.org/10.7202/028054ar

Tous droits réservés @ C Département des relations industrielles de l'Université Laval, 1969
Ce document est protégé par la loi sur le droit d'auteur. L'utilisation des services d'Érudit (y compris la reproduction) est assujettie à sa politique d'utilisation que vous pouvez consulter en ligne.

https://apropos.erudit.org/fr/usagers/politique-dutilisation/ 
il entreprendre la formation des travailleurs, sur le lieu même du travail ? Quelles méthodes et moyens faudrait-il développer pour encourager la formation de tous les travailleurs? Finalement, signalons la valeur méthodologique et empirique des huit études de cas qui amènent les conclusions du professeur Somers. Ces études serviraient facilement toute évaluation de programmes de formation, à condition de développer des variables contrôlées exactes et de n'en pas attendre de réponses qu'elles ne pourraient apporter.

\section{René PARENTEAU.}

Formation professionnelle : plus qu'une préoccupation, une nécessité, Conférence syndicale mondiale sur la formation professionnelle, Turin, Italie, février 1968, 144 pages.

La Conférence Syndicale Mondiale sur la formation professionnelle était l'aboutissement logique des engagements pris par les organismes dirigeants de la Fédération syndicale mondiale, la preuve de l'esprit conséquent avec lequel la plus grande organisation syndicale internationale se préoccupe des problèmes ayant un intérêt vital pour la classe ouvrière mondiale, avec lequel elle s'efforce de réaliser une politique syndicale qui corresponde aux exigences d'une situation générale en évolution permanente.

Sur la base des expériences de leurs centrales respectives, les délégués ont présenté un large éventail des problèmes que pose la formation professionnelle dans les pays de systèmes économiques et sociaux différents. Les pays en voie de développement étaient largement représentés. La discussion a reflété cet état de chose en attribuant une attention particulière aux problèmes et aux besoins de ces pays.

\section{Pierre DIONNE}

Immigrants in Industry, par Sheila Patterson, London, Oxford University Press, 1968, 425 pages.

Cette publication repose principalement sur une imposante enquête faite dans l'industrie londonnienne, et plus particulièrement dans le quartier Croydon, au cours des années 1958-60. Le retard apporté à la publication dépend d'une réorganisation des données.

La publication se divise en quatre parties :

Partie 1. Le cadre de référence. L'auteur nous met en contact avec le lieu de l'enquête et l'enquête elle-même. Le but principal était de chercher dans quelles industries et à quelles sortes de travaux les nouveaux arrivés s'intégraient: quelles étaient leurs aptitudes, leurs qualifications et leurs aspirations; quelles sortes de relations ils établissaient avec leur milieu de travail; jusqu'à quel point ils s'y sont intégrés et quels sont les facteurs de cette intégration.

Partie 2. Le rapport de travail. Cette partie explique la sélection des entreprises, et expose les données recueillies dans tous les genres d'industries et de services. Ces données sont très élaborées.

Partie 3. Résultats et conclusions. L'auteur poursuit son étude en fonction des différents groupes d'immigrants. Elle se penche sur tous les indices des différentes phases de l'intégration industrielle et sur tous les grands facteurs qui accélèrent ou ralentissent cette intégration. Les groupes diffèrent considérablement quant à leur bagage socio-économique et culturel, quant à leurs intentions et autres critères importants. Ce bagage explique souvent leurs objectifs, leurs normes et leurs attitudes.

Partie 4. Etude dans trois établissements spécifiques. L'auteur se penche sur Telelux, Chocolac et Polplastics. Dans chacun des cas l'auteur décrit la situation de l'établissement, puis traite des problèmes de relations industrielles suscités par l'immigration: recrutement, sélection, formation et supervision. De plus, l'auteur fait une analyse assez détaillée de l'intégration des immigrants dans leur milieu de travail (appréciations, promotions, etc.), et dans leur milieu social.

Il convient de souligner l'importance des tableaux qui apparaissent en appendice, illustrant la chaîne d'autorité des établissements Telelux et Polplastics, et quelles places les immigrants y détiennent.

\section{Pierre BRIEN}

